\title{
Synthesis of Cobalt Nano Crystals in Aqueous Media and Its Characterization
}

\author{
Pamela Alex*, Sanjib Majumdar, Jugal Kishor, Inderkumar G. Sharma
}

Materials Processing Division, Bhabha Atomic Research Centre, Trombay, India.

Email: *alexpamela57@yahoo.co.in

Received January $21^{\text {st }}, 2011$; revised March $10^{\text {th }}, 2011$; accepted June $22^{\text {nd }}, 2011$.

\begin{abstract}
The work demonstrates the production of nano crystals of cobalt in bulk quantities in aqueous medium using hydrazine as the reducing agent. Preparation of Co nano powders of 30 - $70 \mathrm{~nm}$ of $99.99 \%$ purity was accomplished from $0.25-2$ $\mathrm{MCOSO}_{4}$ solutions in batch scale of 0.1 to $1 \mathrm{~kg}$. The results of characterization studies using XRD, SEM, TEM indicate the formation of finer particles with increase in concentration of cobalt ions in solution and dominance of fcc cobalt in room temperature reduction. VSM results revealed a higher saturation magnetization of the nano cobalt at $100 \mathrm{~K}$ to be comparable to that of the bulk metal.
\end{abstract}

Keywords: Magnetic Properties, Magnetic Materials, Thermogravimetric Analysis (Tga), Electron Microscopy, X-Ray Diffraction $(X r d)$

\section{Introduction}

The enhanced mechanical, electronic and magnetic properties [1] of metallic nano particles have triggered proposals for applications as high-density magnetic storage devices [2], hall sensors [3], soft magnetic materials exhibiting higher permeability/lower coercivity [4] and heterogeneous catalysts [5]. Transition metallic (Fe, Co, $\mathrm{Ni}$ ) nano particles have found wide application in catalysts, solar energy absorption and magnetic recording [68]. Of the three transition metals that are normally ferromagnetic, the nano particles of pure cobalt probably have special significance in both theory and technology [9-11], because the metal possesses hexagonal closepacked (HCP or $\alpha$-cobalt) structure besides face-centered cubic (FCC or h-cobalt) structure.

Metallic cobalt nanoparticles have been available from wet-phase synthesis methods for more than 50 years [12], today offering good control of product size and shape. The wide range of synthetic, mainly liquid based, preparation methods offers access to cobalt nano particles of primary particle sizes starting from only several nanometers up to submicron sized materials. Applications for cobalt nano powders generally involve their magnetic properties and include catalysts and magnetic recording and in medical sensors and bio medicine as a contrast enhancement agent for magnetic resonance imaging. Cobalt nano powders are also being tested for site spe- cific drug delivery agents for cancer therapies and in coatings, fuel cells, batteries, DNA circuits and chrome replacement in sporting goods, luxury and consumer products [13].

Many methods, such as decomposition of dicobalt octacarbonyls, pulse current electro-deposition, gas vapor condensation, salts reduced by reducing agent $\mathrm{NaBH}_{4}$ or $\mathrm{H}_{2}$, are used in the preparation of cobalt nano particles [10-17]. Cobalt nano particles are also prepared by reduction of cobalt salt in hydrazine alkaline system [18] involving the application of ultrasound initiative and also in ethanol medium [19]. In a recent publication by Kalyan et al. [20], has reported synthesis of nano cobalt from a cobalt hydrazine complex in $1 \mathrm{gm}$ scale. However production of nano sized cobalt powder in aqueous medium using hydrazine as reductant in bulk quantities has not yet been investigated.

Most of the literature refers to preparation of Co nano powders in micro to few gram scale studies only, without mentioning any details of the reproducibility of results in increased batch scales. In this paper, we describe an easy and inexpensive chemical synthesis route in alkaline medium and a moderately high metal concentration is applied. The preparation of pure cobalt nano particles is conducted in a hydrazine alkaline system, at ambient temperature and completed instantaneously without introduction of a nucleator. We predict that the process 
could be applied for production of Co nano powders in bulk quantities. The size and structure of the resultant nano particles were characterized by transmission electron microscopy (TEM), X-ray diffraction (XRD), Scanning electron microscopy (SEM) and Thermo gravimetric analysis. The powder was also subjected to magnetic evaluation.

\section{Experimental Procedure}

Cobalt sulphate, Hydrazine, and Sodium hydroxide used in the experiments were the guaranteed reagent of $\mathrm{E}$. Merck. Typically, an appropriate amount of cobalt sulphate $(0.25 \mathrm{M}$ to $2 \mathrm{M})$ was dissolved directly in dematerialized water. Then, required amount of hydrazine (2 - 4 $\mathrm{M})$ and $\mathrm{NaOH}(0.5$ to $2.5 \mathrm{M})$ was added in sequence. For each experiment, the entire $\mathrm{Co}^{2+}$ ion in the solution was allowed to precipitate. The reduced powder was washed thoroughly with dilute $\mathrm{HCl}$ to remove traces of alkali and finally with ethanol, dried under vacuum, weighed and was stored in propanol solution. Extensive studies were carried out to establish ideal condition of precipitation of cobalt nano powders. The reduction reaction could be expressed as

$$
2 \mathrm{Co}^{2+}+\mathrm{N}_{2} \mathrm{H}_{4}+4 \mathrm{OH}^{-} \rightarrow 2 \mathrm{Co}+\mathrm{N}_{2}+4 \mathrm{H}_{2} \mathrm{O}
$$

$\mathrm{XRD}$ and SEM analysis were done after separating the precipitate from solution using a permanent magnet, then washing the precipitates using ethanol, and finally vacuum drying at room temperature. XRD measurements were performed on a Rigaku D/max III X-ray diffractometer using Mo $K \alpha$ radiation $(\lambda=0.07093 \mathrm{~nm})$. The particle size was measured by the X-Ray Diffraction method using the Scherer Formula. The standard sample of Ceria was taken along with the cobalt sample and the diffraction pattern was obtained. The FWHM, $d$ and the $2 \theta$ were obtained from the diffraction patterns of both the standard and the Co powder. Considering the equation $F W H M^{2}=U \tan ^{2} \theta+W$, the regression curve was plotted between the $F W H M^{2}$ and $\tan ^{2} \theta$ for the standard Ceria sample and the curve fitting was done. The values of the constants $U, V$ and $W$ obtained from that curve fitting were then used to calculate the FWHM $\left(B_{s}\right)$ at different $\theta$ values, at which the peaks were obtained form the Co powder. The actual broadening $(B)$ was calculated using the relationship $B=\left(B_{e}^{2}-B_{s}^{2}\right)^{1 / 2}$, where $B_{e}$ the experimental FWHM value for the cobalt sample, and $B_{s}$ is the calculated FWHM value for the standard. The value of the $B$ so obtained was then put in the Scherer Formula to determine the value of particle size.

SEM analysis was carried out using Camscan MV $2300 \mathrm{CT} / 100$. The particle sizes were determined by TEM using a TITAN model JEM-1200EX at $160 \mathrm{kV}$. The samples for TEM analyses were obtained by diluting the dispersed solution with ethanol and then placing a drop of the diluted solution onto a Formvar-co- vered copper grid and evaporated in air at room temperature. Before the samples were withdrawn, the cobalt nanoparticle-dispersed in ethanol solutions were sonicated for 1 min to obtain the better particle dispersion on the copper grid. Thermo gravimetric analysis of the powder was conducted using Setaram make thermo balance (Model Setsys Evolution). Powder was kept in an alumina crucible and heated in inert atmosphere at about $600^{\circ} \mathrm{C}$. Magnetic studies of the cobalt powder were carried out using Vibrating Sample Magnetometer of Oxford Make.

\section{Results and Discussion}

Concentration of alkali in the cobalt solution played a major role in controlling the precipitation reaction. This was clearly visible in the preliminary experiments we carried out, by using a solution of $1 \mathrm{M} \mathrm{CoSO}_{4}$ and a $\mathrm{pH}$ greater than 12 [21]. As observed, Co forms a complex of $\mathrm{Co}(\mathrm{OH})^{4+}$ with $\mathrm{NaOH}$ and precipitates to form $\mathrm{Co}(\mathrm{OH})_{2}$ subsequently. Our investigations were aimed at selecting the required alkali concentration for the complete precipitation of $\mathrm{Co}$ from high strength Co solutions. Concentration of $\mathrm{NaOH}$ was varied from $0.5 \mathrm{M}-2.5 \mathrm{M}$ (Figure 1), when the color of the cobalt hydroxide precipitate changes from dark pink to bluish violet and finally precipitates as grayish Co powder.

Once, the bulk precipitation is initiated the rate of reduction is fast and completes within few minutes. The reduction fraction in Figure 1 essentially indicates the percentage ratio of precipitated Co metal to starting Co ions. It can be seen that increase in alkali concentration highly accelerates the reduction of Co ions and a com-

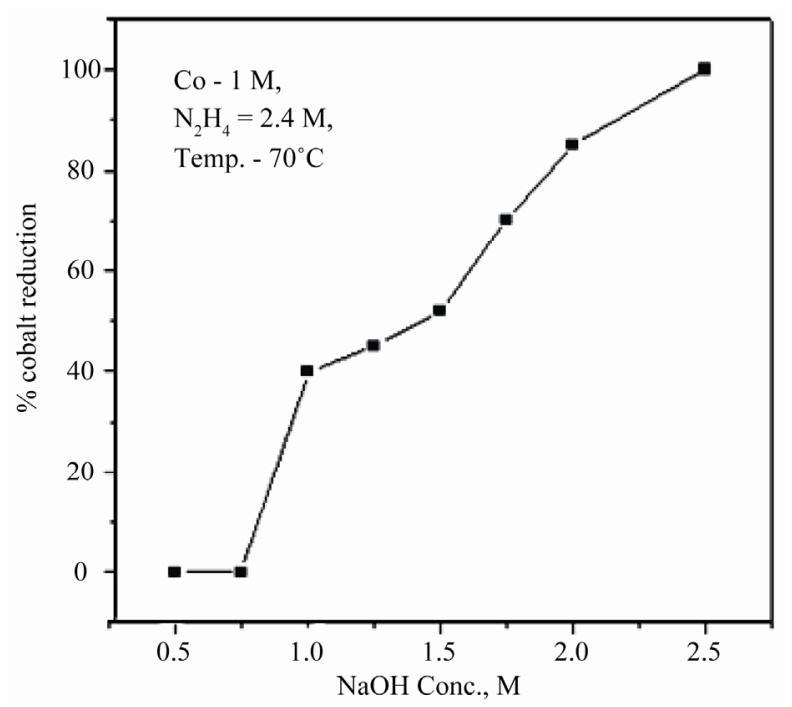

Figure 1. Effect of $\mathrm{NaOH}$ Concentration on reduction of $\mathrm{Co}^{2+}$ to Co. 
plete reduction of Co ions is possible with an effective concentration of $2.4 \mathrm{M} \mathrm{NaOH}$ in the solution.

Figure 2 shows the reduction rate of Co ions with the increase in concentration of hydrazine. The rate increases linearly up to $4 \mathrm{M}$, and becomes constant. Hence a minimum concentration of $4 \mathrm{M} \mathrm{N}_{2} \mathrm{H}_{4}$ was required for the complete precipitation of cobalt ions from the solution. The ratio of Co to hydrazine was maintained at 1:16 for any strength of Co solution.

The $\mathrm{Co}^{2+}$ ions in solution also contributed to the rate of reduction and size of Co nano particles. It was observed that lower concentration of $\mathrm{Co}$ ions in solution exhibited very slow kinetics and finer particles. However when concentration of $\mathrm{Co}$ in solution was increased from 0.25 $\mathrm{M}$ to $2 \mathrm{M}$, the particle size increased up to a Co concentration of $0.5 \mathrm{M}$, and then started giving finer particles at a very fast rate (supported by XRD Figure 4). This anomalous behavior of Co nano powder produced at as high a concentration as that of $1 \mathrm{M}$, can be due to faster nucleation and faster nucleation kinetics of Co particles at higher concentrations and is advantageous in producing nano powders in bulk. However, further increase in Co concentration resulted in severe filtration problems and was discontinued.

The effect of temperature of the reaction on rate of reaction can be seen from Figure 3. The rate of reaction was slow at lower temperatures and increases steadily at temperatures greater than $50^{\circ} \mathrm{C}$ and stabilizes above $70^{\circ} \mathrm{C}$. This is due to the highly exothermic nature of the reduction reaction. At lower temperatures the nucleation time is more and kinetics of the reaction is slow. Increase in temperature and availability of higher surface area with formation of cobalt metal, accelerates the reduction reaction. A temperature of $70^{\circ} \mathrm{C}$ not only increases the reaction rate, when bulk quantities of Co has to be produced,

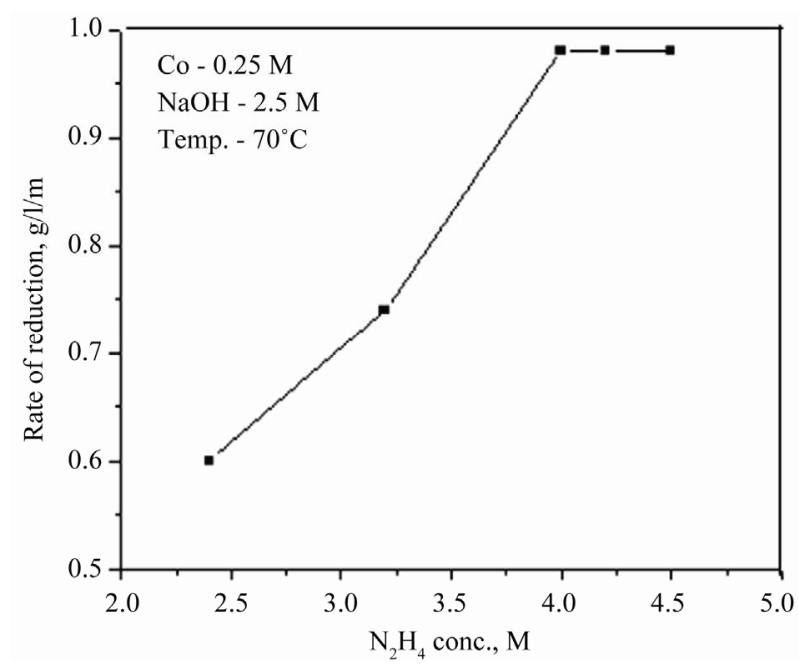

Figure 2. Dependence of Co precipitation on Hydrazine.

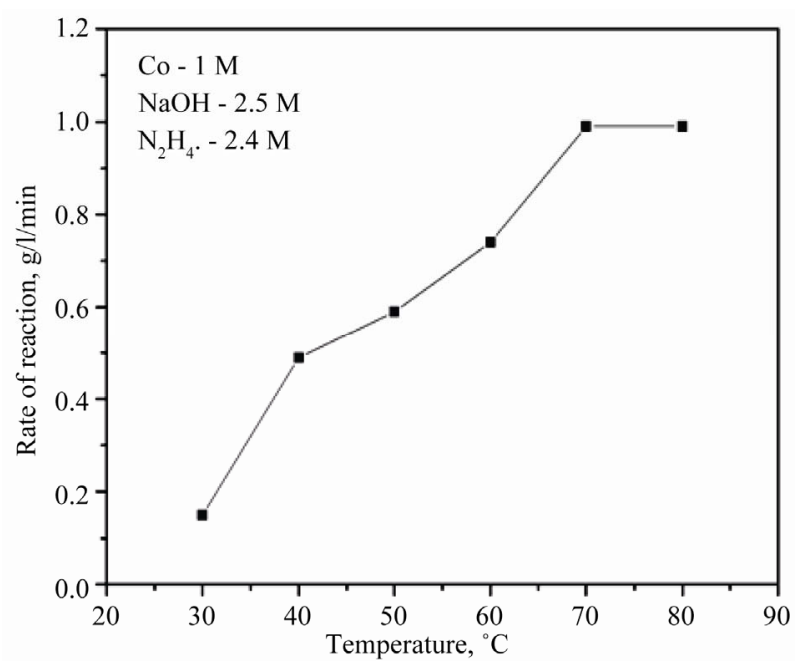

Figure 3. Effect of Temp. on Cobalt reduction.

but also results in much finer particle at as high a concentration as $1 \mathrm{M}$. In fact it requires only 10 minutes preparing Co nano powder in kilogram scale. The production of Co nano powder can be easily accomplished by maintaining a cobalt concentration as high as $1 \mathrm{M}$, at $\mathrm{pH}$ greater than 13 and at a temperature of $70^{\circ} \mathrm{C} .1 \mathrm{~kg}$ of $\mathrm{CoSO}_{4}$ gives roughly $200 \mathrm{~g}$ of Co metal powder.

Most of the reported work on Co nano powder preparation is in $\mathrm{mg}$ or gm scale of Co while we have attempted to make Co nano powder in larger scale. The ease with which the nano powder could be prepared in this investigation can be scaled up to large scale production and its future application in various fields.

\section{Characterization Studies}

The detailed XRD analysis carried out using the Co powder produced at different conditions is presented in Figure 4. Mostly, the peaks from both the hep and fcc phases are detected. It is observed that the peak broadening is more for the Co powder produced using $0.25 \mathrm{M}$ concentration of cobalt solution as compared to that produced using $0.5 \mathrm{M}$ solutions at $70^{\circ} \mathrm{C}$.

This indicates the formation of finer particles at lower cobalt ion concentration. The particle size calculated using Scherrer formula is around $24 \mathrm{~nm}$ and $49 \mathrm{~nm}$, respectively for $0.25 \mathrm{M}$ and $0.5 \mathrm{M}$ solutions. The dominance of one particular phase is also detected in the Co powder produced at room temperature. Figure 5 represents the calculated size of Co particles using the XRD data presented in Figure 4. It is observed from Figure 5 that the cobalt particle size is reduced with increase in the cobalt concentration in the solution beyond $0.5 \mathrm{M}$. This anomalous behavior could be due the faster kinetics of the reduction process with increasing the cobalt ion concentration. The growth of the individual particles is 


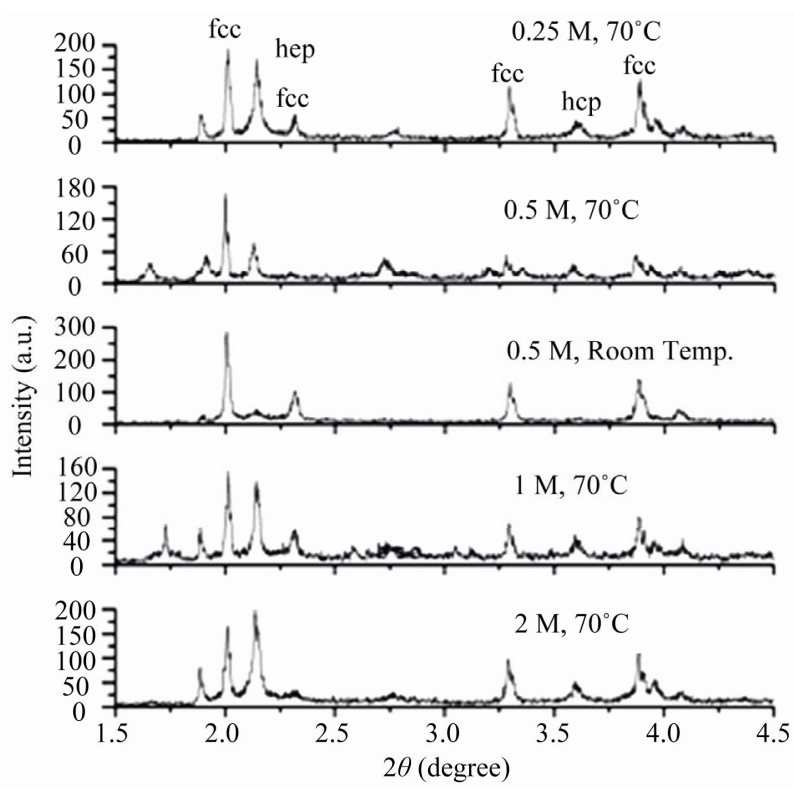

Figure 4. XRD plots of the cobalt nano powder produced using different cobalt concentration and temperature.

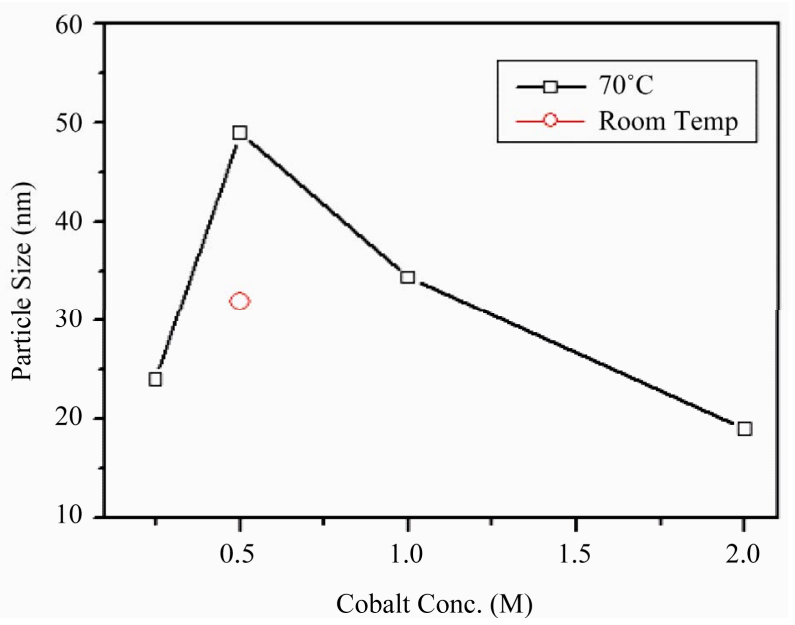

Figure 5. Change in particle size with cobalt concentration obtained from XRD results.

restricted due to the faster reaction kinetics at higher concentrations.

The SEM image of the agglomerated Co particles is presented in Figure 6. The single domain nano size ferromagnetic cobalt particles have the tendency to align in particular direction.

Figure 7 shows the TEM image of some of the particles indicating an average size of around $70 \mathrm{~nm}$. Difficulties were faced to disperse the individual particles due to the strong magnetic characteristics. The diffraction pattern (inset in Figure 7) indicated the presence of both hcp and fcc phases in the powder. The inner ring containing diffraction spot doublets is due to hcp phase

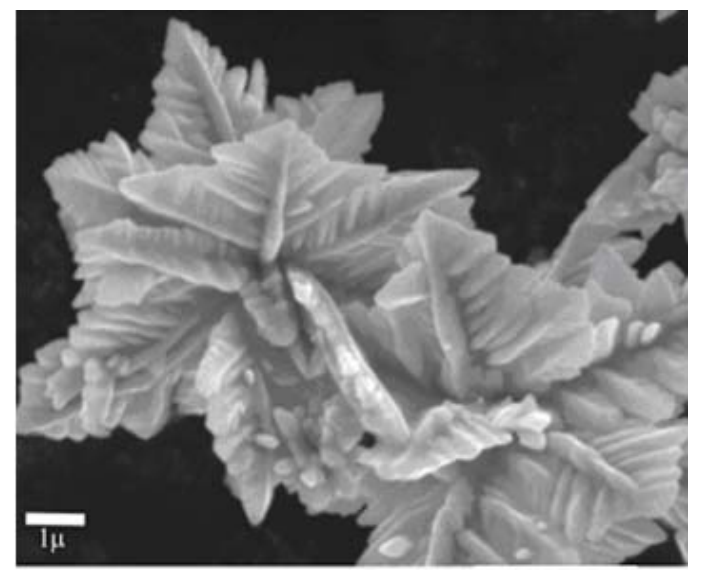

Figure 6. SEM image of the agglomerate showing the alignment of particles forming leaf like structure.

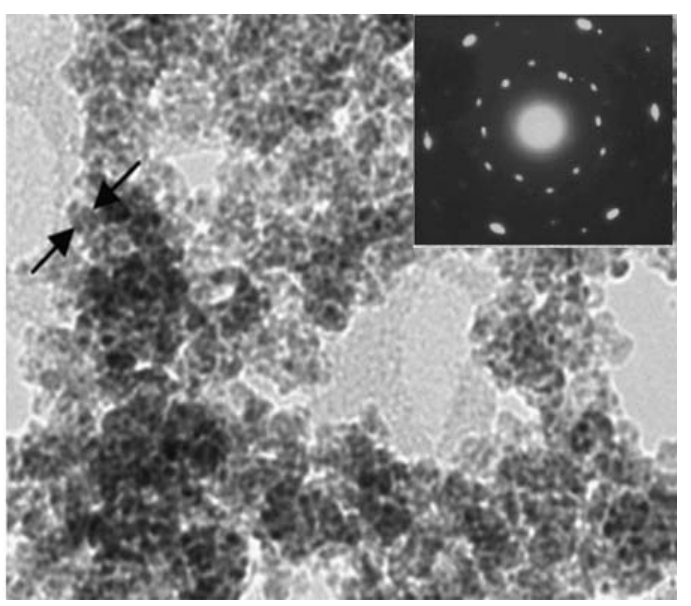

Figure 7. TEM image of the cobalt nano particles; inset SAD pattern confirming both HCP and FCC rings due to nano size.

and the next ring is for the fcc phase. Since, an aqueous process has been adopted to prepare cobalt nano powder, a marginal amount of gases are adsorbed as indicated by slight weight loss during heating in TG studies (Figure 8).

Figure 9 shows the magnetic hysteresis loops of the as prepared Co material recorded at 298 and $100 \mathrm{~K}$. Both loops showed a very small coercivity $(100 \mathrm{Oe})$ together with a high saturation magnetization. While saturation magnetization at $298 \mathrm{~K}$ of $124 \mathrm{emu} \cdot \mathrm{g}^{-1}$ lay below the reference value for bulk cobalt metal $\left(164-165 \mathrm{emu} \cdot \mathrm{g}^{-1}\right)$, at $100 \mathrm{~K}$ the saturation magnetization was within $98 \%$ of reference value $\left(164-165 \mathrm{emu} \cdot \mathrm{g}^{-1}\right)$ [22]. The saturation magnetization at room temperature was enhanced, favoring the use of the present material for magnetic applications. The data at $100 \mathrm{~K}$ suggested a high content of magnetic cobalt $(>85 \%)$. The reduced saturation magnetization at $298 \mathrm{~K}$ indicated a strongly temperature de- 


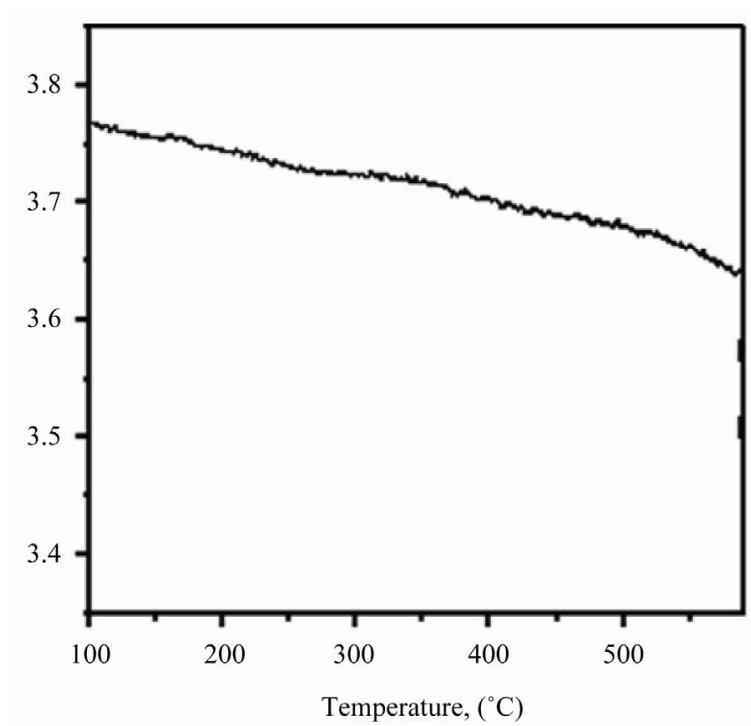

Figure 8. TG plot showing a very little change in weight due to evaporation of adsorbed gases.

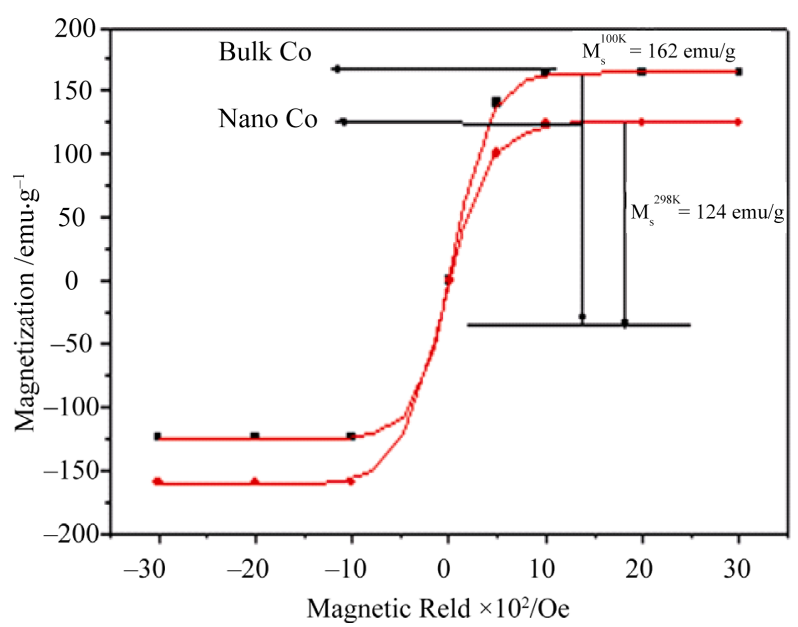

Figure 9. Magnetic hysteresis of as prepared Co nano powder measured at 298 and $100 \mathrm{~K}$ exhibiting small coercivity and a large saturation magnetization (124 - 164 emu $\left.\cdot g^{-1}\right)$.

pendent magnetic response which agrees with prior observations [23]. The low coercivity of $100 \mathrm{Oe}$ is also characteristic of single domain magnetization.

\section{Conclusions}

A detailed study on the preparation of nano size Co powder from $\mathrm{CoSO}_{4}$ salts by varying different parameters indicated that, a hydrazine concentration of $4 \mathrm{M}$ was sufficient to precipitate Co nano powder of $30-70 \mathrm{~nm}$ from highly alkaline cobalt solution of $1 \mathrm{M}$, at a temperature of $70^{\circ} \mathrm{C}$. A reduction in particle size was identified with increase in cobalt ion concentration, which is not reported in earlier findings. Dominance of fcc phase was observed in the nano cobalt particles produced at room temperature. The powder due to its highly ferromagnetic nature was agglomerated and formed a flower like image as visualized in SEM. The TEM analysis indicated a particle size of $50-70 \mathrm{~nm}$ was produced. However, XRD analysis by Scherer formula gave the size to be around $24-49 \mathrm{~nm}$. The magnetic studies revealed a very high content of magnetic cobalt.

\section{Acknowledgements}

The authors wish to thank Dr. Madangopal Krishnan and Dr. R. Tiwari of Material Science Division, BARC, for their support in XRD and TEM analysis.

\section{REFERENCES}

[1] Q. Yan, T. Kim, A. Purkayastha, P. G. Ganesan, M. Shima and G. Ramanath, "Enhanced Chemical Ordering and Coercivity in FePt Alloy Nanoparticles by Sb-Doping," Advanced Materials, Vol. 17, 2005, pp. 2233-2236. doi:10.1002/adma.200500949

[2] G. A. Held and G. Grinstein, "Quantum Limit of Magnetic Recording Density," Applied Physics Letters, Vol. 79, 2001, pp. 1501-1503. doi:10.1063/1.1396317

[3] K. Tetz, L. Pang, and Y. Fainman, "High Resolution Surface Plasmaon Resonance Sensor Based on Linewidth Optimized Nanohole Array Transmittance," Optics Letters, Vol. 31, 2006, pp. 1528-1530. doi:10.1364/OL.31.001528

[4] B.-H. Lee, Y. J. Lee, K. H. Min, D.-G. Kim and Y. D. Kim, "Synthesis of Nano Crystalline Spherical CobaltIron (Co-Fe) Alloy," Materials Letters, Vol. 59, 2005, pp. 3156-3159. doi:10.1016/j.matlet.2005.05.027

[5] A. T. Bell, "The Impact of Nanoscience on Heterogeneous Catalysis" Science, Vol. 299, 2003, pp. 1688-1691.

[6] M. L. Wagner, L. D. Schmidt, Model Catalytic Oxidation Reactions: Oxygen with $\mathrm{H}_{2}, \mathrm{NH}_{3}$, and $\mathrm{N}_{2} \mathrm{H}_{4}$," The Journal of Physical Chemistry, Vol. 99, 1995, pp. 805-815. doi:10.1021/j100002a051

[7] S. W. Moore, "Solar Absorber Selective Paint Research," Solar Energy Materials, Vol. 12, 1985, pp. 435-447. doi:10.1016/0165-1633(85)90037-1

[8] X. G. Li, A. Chiba and S. Takahashi, "Preparation and Magnetic Properties of Ultra Fine Particles of $\mathrm{Fe}-\mathrm{Ni}$ Alloys," Journal of Magnetism and Magnetic Materials, Vol. 170, No. 3, 1997, pp. 339-345.

[9] L. Wei, T. Yang and Y.H. Tang, "Synthesis and Properties of Nano Crystalline Nonferrous Metals Prepared by Flow-Levitation-Molding Method," Transactions of Nonferrous Metals Society of China, Vol. 17, No. 6, 2007, pp. 1347-1351. doi:10.1016/S1003-6326(07)60275-1

[10] W. Gong, H. Li, Z. Zhao and J. Chen, "Synthesis and Magnetic Properties of Ni Nano Crystals," Journal of Applied Physics, Vol. 69, 1991, pp. 5119-5125. doi:10.1063/1.348144 
[11] E. Sowakai, M. Leonowiczi, B. Andrzejewski and G. I. Dzhardimaliev, "A Cobalt Nanoparticles Processed by Thermal Decomposition of Metal-Containing Monomers," Materials Science-Poland, Vol. 24, No. 1-2, 2006, pp. 312-317.

[12] G. N. Glavee, K. J. Klabunde, C. M. Sorensen, G. C. Hadjipanayis, "Sodium Borohydride Reduction of Cobalt Ions in Nonaqueous Media. Formation of ultrafine Particles (Nanoscale) of Cobalt Metal," Inorganic Chemistry, Vol. 32, No. 4, 1993, pp. 474-477.

[13] E. Erb and A. M. El-Sherik, U.S. Patent, No. 5353266, 1994.

[14] J.C. Sanchez-Lopez, A.R. Gonzalez-Elipe, A. Fernandez, "Passivation of Nano Crystalline Al Prepared by the Gas," Journal of Materials Research, Vol. 13, No. 3, March 1998, pp. 703-707.

[15] P. Saravanana, K. Raob, M. Premkumara and A. K. Singha, "Spherical Granular Structures of Ag/Co Nanoparticles: Synthesis, Characterization and Magnetic Properties," Journal of Alloy and Compounds, Vol. 509, 2011, pp. 3880-3885. doi:10.1016/i.jallcom.2010.12.141

[16] K. Simeonidis, S. Mourdikoudis, A. Vilalta-Clemente, I. Tsiaoussis, M. Angelakeris and O. Kalogirou, "Shape and Composition Oriented Synthesis of Cobalt Nanoparticles," Physics of Advanced Materials Winter School, 2008.

[17] B. Q. Xie, Y. T. Qian, S. Y. Zhang, S. Q. Fu and W. C.
Yu, "A Hydrothermal Reduction Route to Single-Crystalline Hexagonal Cobalt Nanowires," European Journal of Inorganic Chemistry, Vol. 12, No. 2, 2006, pp. 2454 2459.

[18] C. P. Gibson and K. J. Putzer, "Synthesis and Charecterization of Anisotropic Co Nano Crystals," Science, Vol. 267, 1995, pp. 1338-1340.

[19] H.-G. Zheng, J. Lianga, J. Zenga and Y. Qiana, "Preparation of Nickel Nanopowders in Ethanol-Water System (EWS)," Materials Research Bulletin, Vol. 36, No. 5-6, 2001, pp. 947-952.

[20] S. S. Kalyan Kamal, P. K. Sahoo, M. Premkumarand and K. Chandra Sekar, "Synthesis of Co Nano Particles by a Modified Polyol Method," Journal of Alloys and Compounds, Vol. 474, 2009, pp. 214-218. doi:10.1016/j.jallcom.2008.06.160

[21] S. S. Djokic, "Electron Microscope Study of the Nucleation and Growth of Electroless Cobalt and Nickel," Journal of Electrochemistry, Vol. 7, July 1997, pp. 144-149.

[22] R. Pauthenet, "Experimental Verification of Spin Wave Theory in High Fields," Journal of Applied Physics, Vol. 53, 1982, pp. 8187-8190. doi:10.1063/1.330287

[23] C. M. Schneider, P. Bressler, P. Schuster, J. Kirschner, J. J. de Miguel, R Miranda and S Ferrer, "Characterization of New Materials by Surface-Sensitive Techniques," Vacuum, Vol. 41, 1990, pp. 503-505. 